
Jul 6.

\title{
Symptoms of anxiety and depression in type 2 diabetes: Associations with clinical diabetes measures and self- management outcomes in the Norwegian HUNT study.
}

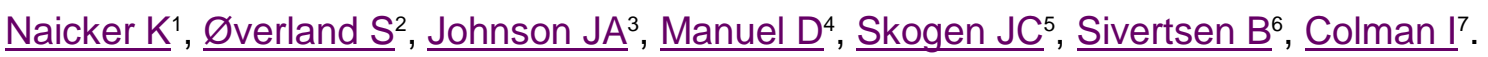

Author information

Abstract

OBJECTIVE:

To determine if symptoms of depression and anxiety are differentially associated with clinical diabetes measures and self-management behaviours in individuals with Type 2 diabetes, and whether these associations vary by patient sex.

RESEARCH DESIGN AND METHODS:

A cross-sectional analysis using data from 2035 adults with Type 2 diabetes in the NordTrøndelag Health Study. Multivariate logistic regression was used to explore associations between symptoms of depression and anxiety and waist girth, HDL cholesterol, systolic blood pressure, triglycerides, c-reactive protein, glycemic control, diet adherence, exercise, glucose monitoring, foot checks for ulcers, and the subjective patient experience. Analyses were stratified by sex.

RESULTS:

Depression was associated with a lower likelihood of avoiding saturated fats (OR=0.20 $[95 \% \mathrm{Cl}$ : $0.06,0.68]$ ) and increased odds of physical inactivity (OR=1.69 [95\% Cl: 1.37, 2.72]). Anxiety was associated with increased odds of eating vegetables ( $\mathrm{OR}=1.66[95 \% \mathrm{Cl}: 1.02,2.73])$, and an over two-fold increase of feeling that having diabetes is difficult. In women, anxiety was associated with elevated c-reactive protein levels ( $\mathrm{OR}=1.57$ [95\% $\mathrm{Cl}: 1.05,2.34])$. In men, depressive symptoms were associated with elevated $\mathrm{HbA1c}(\mathrm{OR}=5.00[95 \% \mathrm{Cl}: 1.15,8.23)$. CONCLUSIONS:

Symptoms of depression and anxiety were differentially associated with some key diabetesrelated measures. Our results suggest sex-specific differences with respect to two important clinical outcomes (i.e., anxiety and CRP in women and depression and glycemic control in men). These findings should alert practitioners to the importance of detection and management of psychological symptoms in individuals with Type 2 diabetes.

Copyright @ 2017. Published by Elsevier Ltd.

KEYWORDS:

Anxiety; Depression; Diabetes self-management; Metabolic control; Type 2 diabetes 
PMID:

28704763

DOI:

10.1016/i.psyneuen.2017.07.002 